\title{
ECCENTRIC CONTRACTIONS INDUCE RAPID ISOMETRIC TORQUE DROP IN DYSTROPHIN-DEFICIENT DOGS
}

\author{
Christopher J. Tegeler, BS ${ }^{1}$, Robert W. Grange, PhD², Daniel J. Bogan, BA $^{3}$, Chad D. \\ Markert, PhD ${ }^{1}$, Doug Case, $\mathrm{PhD}^{4}$, Joe N. Kornegay, DVM, PhD ${ }^{3}$, and Martin K. Childers, DO, \\ $\mathrm{PhD}^{1,5}$ \\ ${ }^{1}$ Wake Forest University Health Sciences, Institute for Regenerative Medicine, 391 Technology \\ Way, Winston-Salem, North Carolina 27101, USA \\ ${ }^{2}$ Department of Human Nutrition, Foods and Exercise, College of Agriculture \& Life Sciences, \\ Virginia Polytechnic Institute and State University, Blacksburg, Virginia 24061, USA \\ ${ }^{3}$ Departments of Pathology and Laboratory Medicine and Neurology, School of Medicine, \\ University of North Carolina-Chapel Hill, Campus Box 7525, Chapel Hill, North Carolina \\ 27599-7525, USA \\ ${ }^{4}$ Biostatistical Sciences, Wake Forest University Health Sciences, Winston-Salem, North Carolina \\ 27101, USA \\ ${ }^{5}$ Department of Neurology, School of Medicine, Wake Forest University Health Sciences, \\ Winston-Salem, North Carolina 27101, USA
}

\section{Abstract}

We tested the hypothesis that eccentric contractions (ECCs) rapidly induce greater-than-normal isometric torque drop in dystrophin-deficient golden retriever muscular dystrophy (GRMD) muscles. ECCs were imposed by forcibly stretching activated muscles. The results indicate that isometric torque drop was greater in GRMD versus controls $(P<0.0001)$. Our findings support the hypothesis that ECCs induce greater-than-normal isometric torque drop in GRMD muscles. The magnitude of ECC-induced isometric torque loss may be an ideal clinical endpoint in the GRMD model.

\section{Keywords}

Duchenne muscular dystrophy; golden retriever muscular dystrophy; stretch; dystrophin; muscle damage

Dogs with golden retriever muscular dystrophy (GRMD) model the phenotype of patients with Duchenne muscular dystrophy (DMD). ${ }^{1}$ In GRMD dogs a splice site mutation ${ }^{2}$ in the dystrophin gene results in progressive muscle weakness, contractures, and gait abnormalities. ${ }^{3,4}$ Many animals succumb within 2 years of onset. Increasing use of the GRMD model in preclinical studies has necessitated development of endpoints to document treatment efficacy. Previously, we reported that eccentric contractions (ECCs) impaired isometric torque in GRMD pelvic limb muscles. This indicates that dystrophin-deficient muscles are highly susceptible to ECC. ${ }^{5}$ In that earlier study we measured isometric torque drop 3 days after ECC. Here we set out to determine if ECC induces rapid torque loss in

(c) 2010 Wiley Periodicals, Inc.

Correspondence to: M.K. Childers; mchilder@wfubmc.edu. 
GRMD muscles. To address this question we measured torque immediately after ECC in GRMD dogs and compared results to carrier and normal controls.

\section{MATERIALS AND METHODS}

Dogs were used and cared for according to principles outlined in the National Institutes of Health Guide for the Care and Use of Laboratory Animals. Newborn GRMD dogs were identified based on elevation of serum creatine kinase ${ }^{6}$ and confirmed with polymerase chain reaction-based genotyping. ${ }^{2}$

\section{Isometric torque Measures}

Anesthetized dogs were positioned in dorsal recumbency. One pelvic limb was immobilized in a stereotactic frame ${ }^{3}$ to align the tibia at a right angle to the femur. To place the cranial tibial muscles at $\mathrm{L}_{0}$ (muscle length at which tetanic torque is maximal) the tibiotarsal joint was positioned at $90^{\circ}$. Adhesive wrap affixed the foot to a pedal mounted on the shaft of a servomotor to measure torque (model 310LR, Aurora Scientific, Aurora, Ontario). Nerve stimulation activated hindlimb muscles of the foot to push (extend) or pull (flex) against the pedal to generate torque. Percutaneous stimulation of the peroneal nerve induced tibiotarsal flexion, whereas tibial nerve stimulation induced tibiotarsal extension. Supramaximal $150 \mathrm{~V}$, $100 \mu$ s pulses were applied (Model S88X stimulator and Model SIU-C isolation unit, Grass Instruments, Quincy, Massachusetts). Tetany was induced by a 1-s train of $50 \mathrm{~Hz}$ pulses. The limb was repositioned and the sequence was repeated. Dynamic Muscle Control computer software (DMC, Aurora Scientific) controlled the servomotor, stimulation timing, and capture of torque responses.

\section{Tibiotarsal ECC Protocol}

Percutaneous peroneal nerve stimulation $(100 \mu$ s square wave pulses, $50 \mathrm{~Hz})$ activated tibiotarsal flexor muscles; stimulating electrodes were positioned until twitches $(\mathrm{Pt})$ reached a maximum. The ECC protocol consisted of an initial isometric contraction followed by a forced stretch imposed by the servomotor (Fig. 1). The servomotor rotated the lever arm $29^{\circ 7}$ opposite to contracting flexor muscles at a rate of $\approx 0.7$ muscle length/s followed by a rapid return to baseline position. During stimulation (100 $\mu$ s square wave pulses over $1 \mathrm{~s}$ at $50 \mathrm{~Hz}$ ) flexor muscles were subjected to $800 \mathrm{~ms}$ isometric and $200 \mathrm{~ms}$ eccentric contractions. This procedure was repeated every $5 \mathrm{~s}$. To avoid fatigue, a 4-min rest followed every 10 contractions and a total of 30 contractions was performed in each animal.

\section{Analysis}

Isometric and eccentric contraction torque profiles were analyzed with Dynamic Muscle Analysis Software (Aurora Scientific). Isometric torque $(\mathrm{N}-\mathrm{m})$ for twitches and tetany were expressed relative to body mass $(\mathrm{kg})$. A Kruskal-Wallis test assessed difference in age among genotypes. Repeated measures analysis of covariance (ANCOVA) assessed genotype differences in maximum torque. An autoregressive covariance structure modeled correlation within animals. Genotype by contraction number interaction tested the significance of genotype versus change in maximum torque across contractions. Contrasts assessed difference in genotypes after contractions and a Bonferroni correction accounted for multiple comparisons. Values were considered statistically different when $P<0.05$.

\section{RESULTS}

\section{Isometric Torque}

GRMD dogs generate lower tibiotarsal flexion and extension tetanic torque compared to normal or carrier dogs $(1.24 \pm 0.08$ vs. $3.60 \pm 0.51$ and $3.46 \pm 0.25$ and $4.12 \pm 0.21$ vs. 7.21 
\pm 0.28 and $5.95 \pm 0.54 \mathrm{~N} \cdot \mathrm{m} / \mathrm{kg}$ body mass, respectively ( $P<0.01$ for each test; Fig. $1 \mathrm{~A}-\mathrm{C})$. No significant differences were detected in tetanic torque values between normal or carrier dogs. The mean age in the affected, normal, and carrier groups was 32.4, 11.7, and 25.9 months, respectively $(P=0.012)$. Age was not significantly associated with either initial torque or ECC-induced torque drop.

\section{ECC-Induced Torque Drop}

Eccentric contractions rapidly induce torque drop to a greater extent in GRMD flexor muscles compared to either normal or carrier muscles $(-63 \pm 4 ;-8 \pm 3$, and $-13 \pm 1 \%$, respectively; $P<0.0001$; Fig. 1D-F). The magnitude of ECC-induced torque drop in GRMD dogs differed significantly from normal and carrier dogs, but normal and carrier dogs did not differ significantly from each other. Differences between genotypes were significant for the fifth and all subsequent contractions (Fig. 1E).

\section{DISCUSSION}

Activities that engage repeated ECC characteristically result in muscle damage accompanied by delayed-onset muscle soreness, increased stiffness, and prolonged loss of strength. ${ }^{8-12}$ Impaired force production after ECC is considered "one of the most valid and unfailing indirect measures of muscle damage." 13 Maximal isometric force drop occurs 3-5 days after contraction-induced damage, probably due to a combination of physical disruption and impaired excitation-contraction coupling. ${ }^{14,15}$ Susceptibility to ECC-induced muscle damage is accentuated by loss of dystrophin in GRMD ${ }^{5,16}$ and, by extension, in DMD. This idea is supported by our previous data, where GRMD dogs experienced marked strength loss 3 days after ECC. ${ }^{5}$ Here we demonstrate that torque drop ensues immediately following ECC. Moreover, most torque loss occurred after 10 ECC (Fig. 1E). Due to the variable phenotype in the GRMD dog, ${ }^{17}$ the magnitude of isometric torque can vary depending on the age of the dog and the selection of muscle groups. Therefore, interpretation of treatment efficacy using isometric measures alone becomes problematic in this model. ${ }^{18}$ In contrast, temporal decrements in torque following ECC provide additional information about muscle function that may provide a robust clinical endpoint for the assessment of therapeutic interventions in the GRMD model.

\section{Abbreviations}

$\begin{array}{ll}\text { ANCOVA } & \text { analysis of covariance } \\ \text { DMD } & \text { Duchenne muscular dystrophy } \\ \text { ECC } & \text { eccentric contractions } \\ \text { GRMD } & \text { golden retriever }\end{array}$

\section{REFERENCES}

1. Cooper BJ, Winand NJ, Stedman H, Valentine BA, Hoffman EP, Kunkel LM, et al. The homologue of the Duchenne locus is defective in X-linked muscular dystrophy of dogs. Nature. 1988; 334:154156. [PubMed: 3290691]

2. Sharp NJ, Kornegay JN, Van Camp SD, Herbstreith MH, Secore SL, Kettle S, et al. An error in dystrophin mRNA processing in golden retriever muscular dystrophy, an animal homologue of Duchenne muscular dystrophy. Genomics. 1992; 13:115-121. [PubMed: 1577476]

3. Kornegay JN, Bogan DJ, Bogan JR, Childers MK, Cundiff DD, Petroski GF, et al. Contraction force generated by tarsal joint flexion and extension in dogs with golden retriever muscular dystrophy. J Neurol Sci. 1999; 166:115-121. [PubMed: 10475104] 
4. Kornegay JN, Tuler SM, Miller DM, Levesque DC. Muscular dystrophy in a litter of golden retriever dogs. Muscle Nerve. 1988; 11:1056-1064. [PubMed: 3185600]

5. Childers MK, Okamura CS, Bogan DJ, Bogan JR, Petroski GF, McDonald K, et al. Eccentric contraction injury in dystrophic canine muscle. Arch Phys Med Rehabil. 2002; 83:1572-1578. [PubMed: 12422328]

6. Valentine BA, Winand NJ, Pradhan D, Moise NS, de Lahunta A, Kornegay JN, et al. Canine Xlinked muscular dystrophy as an animal model of Duchenne muscular dystrophy: a review. Am J Med Genet. 1992; 42:352-356. [PubMed: 1536178]

7. Marsh AP, Eggebeen JD, Kornegay JN, Markert CD, Childers MK. Kinematics of gait in golden retriever muscular dystrophy. Neuromuscul Disord. 2010; 20:16-20. [PubMed: 19932618]

8. Allen DG. Eccentric muscle damage: mechanisms of early reduction of force. Acta Physiol Scand. 2001; 171:311-319. [PubMed: 11412143]

9. Clarkson PM, Newham DJ. Associations between muscle soreness, damage, and fatigue. Adv Exp Med Biol. 1995; 384:457-469. [PubMed: 8585472]

10. Friden J, Lieber RL. Eccentric exercise-induced injuries to contractile and cytoskeletal muscle fibre components. Acta Physiol Scand. 2001; 171:321-326. [PubMed: 11412144]

11. Miles MP, Clarkson PM. Exercise-induced muscle pain, soreness, and cramps. J Sports Med Phys Fitness. 1994; 34:203-216. [PubMed: 7830383]

12. Proske U, Allen TJ. Damage to skeletal muscle from eccentric exercise. Exerc Sport Sci Rev. 2005; 33:98-104. [PubMed: 15821431]

13. Warren GL, Lowe DA, Armstrong RB. Measurement tools used in the study of eccentric contraction-induced injury. Sports Med. 1999; 27:43-59. [PubMed: 10028132]

14. Warren GL, Ingalls CP, Lowe DA, Armstrong RB. Excitation-contraction uncoupling: major role in contraction-induced muscle injury. Exerc Sport Sci Rev. 2001; 29:82-87. [PubMed: 11337828]

15. McHugh MP. Recent advances in the understanding of the repeated bout effect: the protective effect against muscle damage from a single bout of eccentric exercise. Scand J Med Sci Sports. 2003; 13:88-97. [PubMed: 12641640]

16. Childers MK, Staley JT, Kornegay JN, McDonald KS. Skinned single fibers from normal and dystrophin-deficient dogs incur comparable stretch-induced force deficits. Muscle Nerve. 2005; 31:768-771. [PubMed: 15736272]

17. Ambrosio CE, Valadares MC, Zucconi E, Cabral R, Pearson PL, Gaiad TP, et al. Ringo, a golden retriever muscular dystrophy (GRMD) dog with absent dystrophin but normal strength. Neuromuscul Disord. 2008; 18:892-893. [PubMed: 18667316]

18. Liu JM, Okamura CS, Bogan DJ, Bogan JR, Childers MK, Kornegay JN. Effects of prednisone in canine muscular dystrophy. Muscle Nerve. 2004; 30:767-773. [PubMed: 15468337] 
A.

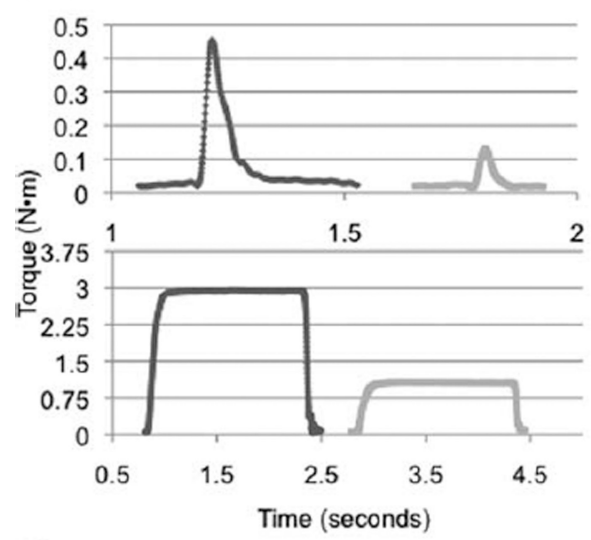

D.

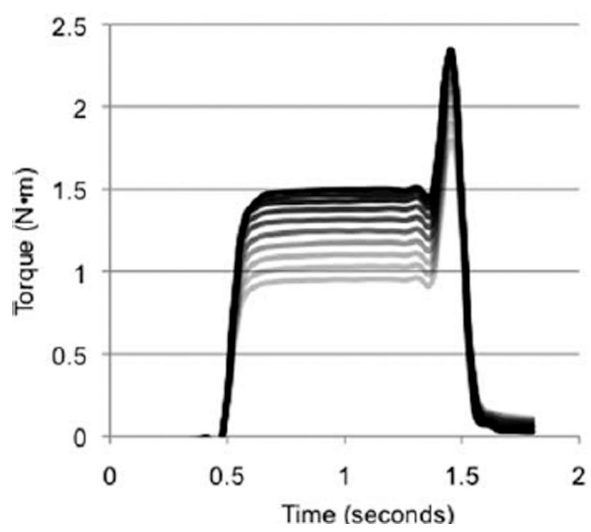

B.

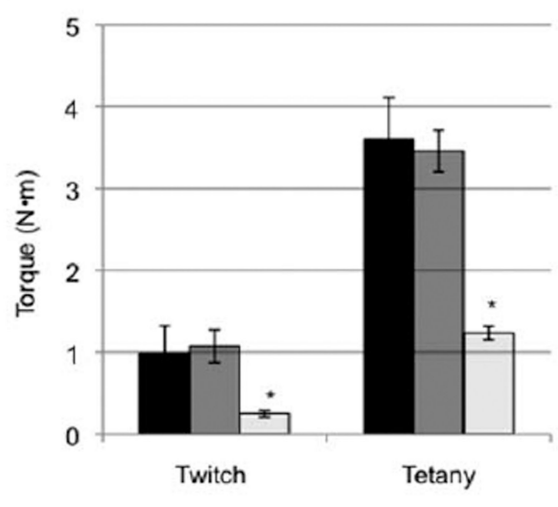

E.

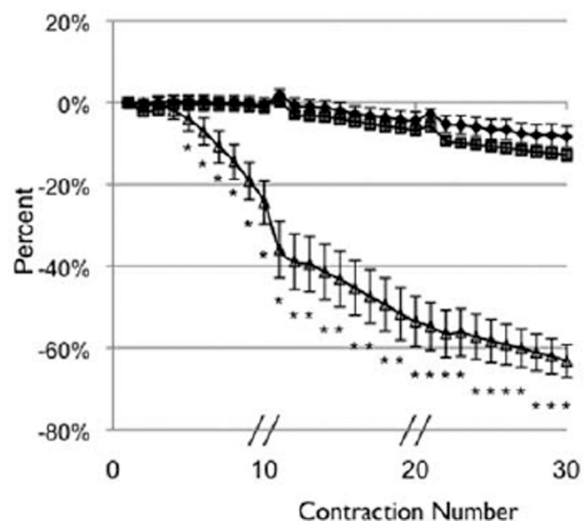

C.

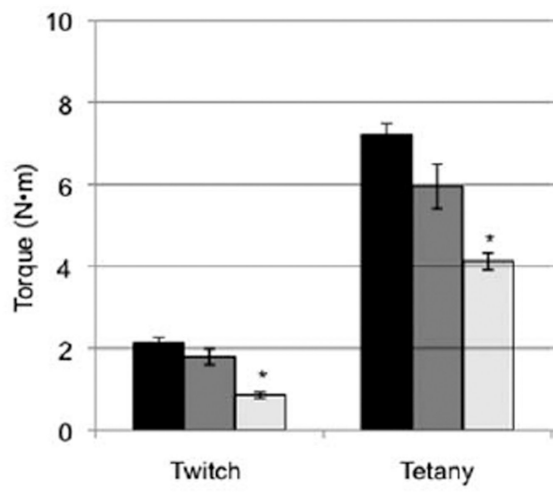

F.

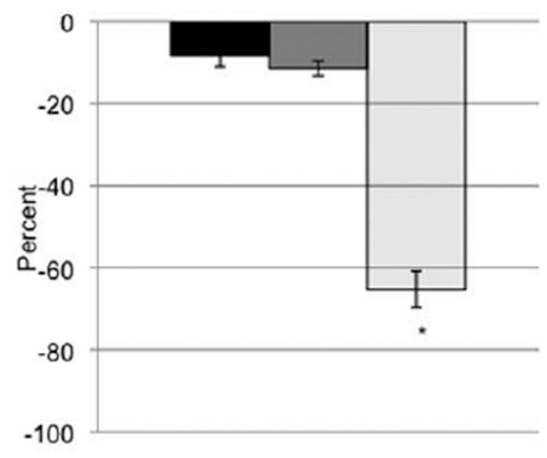

FIGURE 1.

Dynamic force analysis of GRMD $(n=10)$, carrier $(n=7)$, and normal $(n=5)$ dogs. Representative twitch (top panel) and tetany (bottom panel) tracings from normal and GRMD dogs, respectively (A). Compared to normal and carrier dogs, affected GRMD dogs demonstrate lower twitch and tetanic torque values in tibiotarsal flexion (B) and extension (C). Note the y-axis scale. Representative torque tracings (D) during 10 ECCs. The darkest line displays the first contraction while the lightest line shows the tenth contraction.

Following ECC, affected GRMD dogs rapidly lose strength as assessed by isometric torque. Temporal decrements in torque following eccentric injury (E). GRMD dogs display significantly greater-than-normal torque drop after the fifth ECC. Contractions were applied every $5 \mathrm{~s}$. Broken lines through the $\mathrm{x}$-axis indicate a 4-min rest period. Following completion of 30 ECCs, isometric torque varies among genotypes (F). GRMD dogs lose $63 \%$ of their original torque, whereas carriers lose $13 \%$ and normals lose $8 \%$. Black $=$ normal; gray = carrier; light $=$ GRMD. ${ }^{*}$ Significantly different (one-way ANCOVA) compared to the other genotypes $(P<0.05)$. 